\title{
Cystatin C Rescues Degenerating Neurons in a Cystatin B-Knockout Mouse Model of Progressive Myoclonus Epilepsy
}

\author{
Gurjinder Kaur, ${ }^{*}$ Panaiyur Mohan, * \\ Monika Pawlik, ${ }^{*}$ Steven DeRosa, ${ }^{*}$ Jay Fajiculay, ${ }^{*}$ \\ Shaoli Che, ${ }^{*}$ Anders Grubb, ${ }^{\dagger}$ \\ Stephen D. Ginsberg, ${ }^{* \ddagger \S}$ Ralph A. Nixon, ${ }^{\star \neq \pi}$ \\ and Efrat Levy ${ }^{\star \star \|}$ \\ From the Nathan S. Kline Institute," Orangeburg, New York; \\ the Department of Clinical Chemistry, ${ }^{\dagger}$ University Hospital, \\ Lund, Sweden, and the Departments of Psychiatry, ${ }^{\ddagger}$ Physiology \\ and Neuroscience, ${ }^{\S}$ Cell Biology," and Pharmacology," New York \\ University School of Medicine, New York, New York
}

In vitro studies have shown that cystatin $\mathrm{C}$ (CysC) is neuroprotective. Here we demonstrate that $\mathrm{CysC}$ is neuroprotective in vivo, in a mouse model of the inherited neurodegenerative disorder, progressive myoclonic epilepsy type 1 (EPM1). Loss-of-function mutations in the cystatin $B$ (CysB) gene, an intracellular cysteine protease inhibitor, lead to this human disease. A CysB-knockout (CysBKO) mouse model develops symptoms that mimic EPM1. CysB deficiency in these mice results in enhanced cathepsin $B$ and $D$ activities, indicating lysosomal dysfunction. We show that expression of CysC is enhanced in the brains of CysBKO mice. Crossbreeding of CysBKO mice with either CysC-overexpressing transgenic mice or CysCknockout mice demonstrates that clinical symptoms and neuropathologies, including motor coordination disorder, cerebellar atrophy, neuronal loss in the cerebellum and cerebral cortex, and gliosis caused by CysB deficiency, are rescued by $\mathrm{CysC}$ overexpression and exacerbated by CysC deficiency. Thus, CysC effectively rescues the CysB loss-of-function mutations, facilitating the reversal of pathophysiological changes and suggesting a novel therapeutic intervention for patients with EPM1 and other neurodegenerative disorders. (Am J Pathol 2010, 177:2256-2267; DOI: 10.2353/ajpath.2010.100461)

Progressive myoclonus epilepsies are genetic disorders characterized by myoclonic and tonic-clonic seizures and age-associated neurological deficits. UnverrichtLundborg disease (EPM1), the most common form of progressive myoclonus epilepsy, is a rare autosomal disorder caused by loss-of-function mutations in the gene encoding cystatin B (CysB). ${ }^{1-3}$ EPM1 has an onset of symptoms at 6 to 15 years of age, and progression with age leads to myoclonic and tonic-clonic seizures, ${ }^{4,5}$ neurological decline, and severe ataxia. ${ }^{4,6}$

CysB, a member of the cystatin family 1 of cysteine protease inhibitors, is mainly found in the cytosol and at lower levels in body fluids. ${ }^{7}$ Cystatin C (CysC), a secreted cysteine protease inhibitor belonging to the type 2 family of cystatins, is found in all mammalian body fluids and tissues. Both CysB and CysC inhibit cathepsins (Cat) B, $\mathrm{H}, \mathrm{L}, \mathrm{S}$, and $\mathrm{K}^{8-10}$ Uncontrolled proteolysis as a result of imbalance between active proteases and their endogenous inhibitors has been associated with diverse diseases such as Alzheimer's disease, cancer, rheumatoid arthritis, multiple sclerosis, muscular dystrophy, liver disorders, Iysosomal disorders, and diabetes (reviewed in Tizon and Levy ${ }^{11}$ ). Furthermore, precursor forms of lysosomal enzymes, which are not susceptible to inhibition by endogenous inhibitors, have leaked from the lysosomes in many diseases. ${ }^{12-14}$ Accordingly, enhanced cytosolic activity of cathepsins due to loss of CysB function may account for the pathology seen in EPM1. ${ }^{15-17}$

Notably, CysC has been implicated in the processes of neuronal repair of the nervous system. Enhanced CysC expression was observed in response to injury, including transient forebrain ischemia, ${ }^{18,19}$ seizures, ${ }^{20}$ and induction of epilepsy. ${ }^{20-22}$ Oxidative stress also stimulates an increase in $\mathrm{Cys} C$ expression in cultured rat primary neurons ${ }^{23}$ and in cerebral microvascular smooth muscle cells in vitro. ${ }^{24}$ Furthermore, we have shown that human CysC is protective in neuronal cultures exposed to a myriad of cy-

Supported by National Institute on Aging (AG017617 to R.A.N.), National Institute of Neurological Disorders and Stroke (NS42029 to E.L.), and Alzheimer's Association (IIRG-07-59699 to E.L.).

Accepted for publication June 22, 2010.

None of the authors disclosed any relevant financial relationships.

Address reprint requests to Efrat Levy, Ph.D., Nathan S. Kline Institute, 140 Old Orangeburg Rd, Orangeburg, NY 10962. E-mail: elevy@nki.rfmh.org. 
totoxic challenges relevant to Alzheimer's disease and neurodegeneration, including nutritional deprivation, colchicine, staurosporine, oxidative stress, and amyloid $\beta(A \beta){ }^{25,26}$

$A$ mouse model for EPM1 has been generated by knocking out the CysB gene. ${ }^{27}$ CysB-knockout (CysBKO) mice develop myoclonic seizures and ataxia, starting before 2 months of age, similar to symptoms seen in the human disease. ${ }^{27}$ Degeneration of cerebellar granule cells, ${ }^{27}$ hippocampal neurons, and cells within the entorhinal cortex was observed in the developing brains of CysBKO mice. ${ }^{28}$ These mice also show gliosis and increased expression of apoptotic and glial activation genes. ${ }^{29}$ The progressive cerebellar atrophy caused by CysB deficiency implicates a required role for CysB expression in the development of the cerebellum. ${ }^{28,30}$

In the present study, we have tested whether CysC protects in vivo against the deleterious effects of CysB deficiency. Using quantitative PCR (qPCR), Western blot, and immunohistochemical analyses, we demonstrated enhanced CysC expression in brains of CysBKO mice, suggesting that CysC-increased expression could counteract CysB deficiency. Crossbreeding CysC overexpressing mice ${ }^{31}$ with CysBKO mice prevented the increase in cathepsin expression and rescued the clinical and neuronal pathologies characterizing loss-of-function of CysB, including loss of motor coordination, cerebellar atrophy, neuronal cell loss in the cerebellum and cerebral cortex, and gliosis. Moreover, the neuropathology induced by CysB deficiency was exacerbated in mice lacking both the $\mathrm{CysB}$ and $\mathrm{CysC}$ genes. These studies underscore the prospective therapeutic use of CysC in EPMI and have implications for other progressive neurodegenerative disorders where lysosomal proteases are dysfunctional.

\section{Materials and Methods}

\section{Animals}

A breeding colony of CysBKO mice was kindly provided by Dr. Richard M. Myers. ${ }^{27}$ Homozygous CysBKO mice were crossed with either transgenic CysC overexpressing mice ${ }^{31}$ (CysC) or homozygous CysC knockout mice ${ }^{32}$ (CysCKO) all on C57BL/6-129Sv background. Homozygous single and double knockout mice were studied together with their littermate and age-matched singly transgenic or nontransgenic controls at 2, 4, and 12 months of age. A similar number of females and males was used for all analyses. No sex differences were observed in any of the tests. The mice were tested for pathological abnormalities, and body weights and brain weights were measured. All animal procedures were performed following the National Institutes of Health guidelines with approval from the Institutional Animal Care and Use Committee at the Nathan S. Kline Institute for Psychiatric Research. All efforts have been made to minimize animal suffering and the numbers of mice used.

\section{Motor Coordination and Balance Measurements}

Motor coordination and balance were evaluated on a rotating rod apparatus (Accuscan Instruments, Colum- bus, $\mathrm{OH}$ ) at 0 and $2 \mathrm{rpm}$ speeds. The mice were subjected to the rotorod for still and rotating experiments for five consecutive trials of 1 minute duration each. The loss in motor coordination and balance of the mice were judged by the time the mice spent on the still or rotating rod. ${ }^{27}$

\section{Immunohistochemistry and Niss/ Staining}

Mice were deeply anesthetized and transcardially perfused with $0.1 \mathrm{M}$ phosphate buffer, $\mathrm{pH} 7.4$ (PBS), followed by $4 \%$ paraformaldehyde in PBS. Brains were postfixed for 12 hours in $4 \%$ paraformaldehyde in PBS at $4^{\circ} \mathrm{C}$ and transferred to PBS. Blocks of 20 formalin-fixed mouse brains were prepared by Neuro Science Associates (Knoxville, TN) and serial sagittal sections $(40 \mu \mathrm{m})$ of the entire extent of the brain were cut. Free-floating sections from all of the mouse groups were then concurrently processed for histological and immunohistochemical examination. Immunohistochemistry was performed as previously described. ${ }^{33}$ Control sections were processed with the omission of the primary or secondary antibodies to exclude nonspecific reactions. Brain sections were stained with Neurotrace Nissl stain (Invitrogen Corporation, Carlsbad, CA) to stain neurons and glia in the tissue. Total area of the cerebellum was measured on five to six sections per mouse, per genotype $(n=5)$, using $\mathrm{NIH}$ ImageJ software (http://rsbweb.nih.gov/ij/, last accessed September 20, 2010).

\section{Western Blot Analysis}

Mice were euthanized, brains were dissected, and the cerebral cortices and cerebella were flash frozen on dry ice. Tissues were homogenized in lysis buffer: $20 \mathrm{mmol} / \mathrm{L}$ Tris- $\mathrm{HCl}$ (pH 7.4), $250 \mathrm{mmol} / \mathrm{L}$ sucrose, $1 \mathrm{mmol} / \mathrm{L}$ EDTA, and $1 \mathrm{mmol} / \mathrm{L}$ EGTA with protease inhibitors as described previously. ${ }^{33}$ Protein concentration was determined by bicinchoninic acid Protein Assay Kit (Pierce, Rockford, $\mathrm{IL}$ ). Equal amounts (30 to $70 \mu \mathrm{g}$ ) of total proteins were boiled in sample buffer (1\% SDS, 3\% glycerol, $1.5 \%$ $\beta$-mercaptoethanol, and $20 \mathrm{mmol} / \mathrm{L}$ Tris- $\mathrm{HCl}, \mathrm{pH} \mathrm{6.8)}$ and separated by $15 \%$ Tris-glycine gel electrophoresis. The proteins were electrophoretically transferred onto a 0.2 um nitrocellulose membrane (Bio Rad, Hercules, CA) in $2.5 \mathrm{mmol} / \mathrm{L} \mathrm{Tris} / 19.2 \mathrm{mmol} / \mathrm{L}$ Glycine $/ 20 \%$ methanol transfer buffer. The membrane was blocked in $5 \%$ milk (Bio Rad) or $5 \%$ bovine serum albumin in $10 \mathrm{mmol} / \mathrm{L}$ Tris, $150 \mathrm{mmol} / \mathrm{L}$ sodium chloride, $\mathrm{pH} 7.5,0.1 \%$ Tween-20, incubated with primary antibody overnight at $4^{\circ} \mathrm{C}$, and with secondary antibody for 1 to 2 hours at room temperature. The membranes were incubated in chemiluminescent fluid (Pierce) for 5 minutes, chemiluminescence was visualized on Reflection Autoradiography film, and the intensity of the bands was quantified. $\beta$-tubulin or $\beta$-actin was blotted on the same membrane as an internal control for loading. The protein bands were scanned, optical density was calculated by using ImageJ, and the ratio of protein intensity to either $\beta$-tubulin or $\beta$-actin in the same lane was calculated. Mean and SEM were calculated for four separate experiments. 


\section{Antibodies Used for Immunofluorescence and for Western Blotting}

The following antibodies were used: rabbit anti-CysC polyclonal antibody (1:1500 for immunofluorescence [ICC] and 1:5000 for Western blotting [WB]; Upstate Biotechnology, Billerica, MA), rabbit anti-Cat $D$ polyclonal antibody (1:500 for ICC and 1:2000 for WB; Scripps, San Diego, CA), rabbit anti-Cat B polyclonal antibody (1:200 for ICC and 1:1000 for WB; Millipore Corporation, Billerica, MA), rabbit anti-glial fibrillary acidic protein (GFAP) polyclonal antibody (1:500 for ICC and 1:5000 for WB; Dako, Carpinteria, CA), mouse anti-neuronal nuclei (NeuN) monoclonal antibody (1:200 for ICC and 1:1000 for WB; Millipore), mouse anti- $\beta$-tubulin monoclonal antibody (1:10000 for WB; Sigma, St. Louis, MO), or mouse anti- $\beta$-actin monoclonal antibody (1:5000 for WB; Abcam, Cambridge, MA), affinity purified rabbit anti-rat CysB antibody, which was raised in our laboratory (Yang et al, manuscript submitted for publication; dilution 1:10 for ICC and 1:100 for WB), and rat anti-Lamp-2 polyclonal antibody (1:5 for ICC; Hybridoma Bank, IA). The following secondary antibodies were used: fluorescence-conjugated anti-rabbit/anti-mouse antibody for ICC (Invitrogen Corporation); horseradish peroxidase conjugated antirabbit and mouse antibodies for WB (GE Health care, Pittsburgh, PA).

\section{Cathepsins B and D Activity Assays}

The cerebral cortices and cerebella were homogenized in lysis buffer without protease inhibitors at $\mathrm{pH}$ 7.0. Homogenates were acidified at $\mathrm{pH} 5.5$, and Cat B was assayed by measuring the release of 7-amino-4-methylcoumarin (amc) from Z-Arg-Arg-amc as described. ${ }^{34}$ Assays were performed in white micro plates in a volume of $200 \mu \mathrm{l}$ mixture (1 to $5 \mu \mathrm{l}$ of enzyme or lysate; $50 \mu \mathrm{l}$ of $0.1 \%$ Brij-35; and $145 \mu$ l of $0.1 \mathrm{M}$ sodium phosphate buffer $\mathrm{pH}$ 6.0 containing $2 \mathrm{mmol} / \mathrm{L}$ EDTA and dithiothreitol; 1 $\mathrm{mmol} / \mathrm{L}$ phenylmethylsulfonyl fluoride, $5 \mu \mathrm{mol} / \mathrm{L}$ pepstatin $\mathrm{A}$, and $5 \mathrm{mmol} / \mathrm{L}$ Z-Arg-Arg-amc). Fluorescence of amc released after 2 hours was read in a Wallac Victor-2 spectrofluorimetric plate reader with a filter set optimized for detection of 4-methyl-7-aminocoumarin (-amc) standard solution with excitation at $365 \mathrm{~nm}$ and emission at $440 \mathrm{~nm}$. Enzyme activity was expressed as the amount of amc released per hour per milligram protein.

Cat $\mathrm{D}$ was assayed at $37^{\circ} \mathrm{C}$ at $\mathrm{pH} 4.0$ by measuring the release of amc containing peptide, 7-methoxycoumarin-4acetyl-Gly- Lys-Pro-lle-Leu-Phe from 7-methoxycoumarin-4acetyl-Gly-Lys-Pro-lle-Leu-Phe-Phe-Arg-Leu-Lys(Dnp)-DArg-NH2 (BioMol-Enzo, Plymouth Reading, PA), as previously described. ${ }^{35}$ Assays were performed in white microplates in a total volume of $100 \mu$ l with or without $3 \mu \mathrm{g}$ of pepstatin for 1 hour. Fluorescence released was read in a Wallac Victor-2 Spectrofluorimetric plate reader with a filter optimized for detection of amc standard solution with excitation at $365 \mathrm{~nm}$ and emission at $440 \mathrm{~nm}$. However, instead of using amc standard, a quenched standard 7-methoxycoumarin-4-acetyl-Pro-Leu-OH was used for express- ing enzyme activity to account for the release of peptide containing amc instead of free amc. Enzyme activity was expressed as the relative amount of quenched standard released per hour per milligram protein.

\section{Real Time qPCR}

QPCR was performed in triplicate on frozen tissue samples dissected from the cerebellum and cerebral cortex of wild type (WT) and CysBKO mice at 9 to 10 months of age. Samples were assayed on a qPCR cycler $(7900 \mathrm{HT}$, Applied Biosystems, Foster City, CA). Mouse TaqMan hydrolysis probes designed for CysB (Mn00432770), CysC (Mn01273163), Cat B (Mm01310507), Cat D (Mn00515586), GFAP (Mn01253033), and glyceraldehyde-3 phosphate dehydrogenase (GAPDH; Mm99999915) were used (Applied Biosystems) as described previously. ${ }^{36-38}$ Standard curves and cycle threshold were generated by using standards obtained from total mouse brain RNA. The delta delta cycle threshold method was used to determine relative gene level differences, and expression levels were presented as percentage of WT as described previously. ${ }^{36,37}$ Negative controls were used for each assay and consisted of the reaction mixture without input RNA.

\section{Statistical Analyses}

Data are presented as mean \pm SD. One way analysis of variance followed by posthoc multiple comparison Bonferroni's tests was used to evaluate the differences between genotypes. As the major objective of the study was to examine whether CysC overexpression/deletion in CysBKO mice alleviate/exacerbate the pathologies induced by CysB deletion, statistical comparisons were made between CysBKO mice and each of the other groups. Behavioral trial results are presented as mean \pm SEM with different time intervals analyzed by using twoway analysis of variance followed by posthoc multiple comparison Bonferroni's tests. Unpaired, two-tailed Student's $t$-test was used to evaluate PCR results because of the limited number of groups.

\section{Results}

\section{Effect of CysC Overexpression on Motor Coordination in CysBKO Mice}

CysBKO mice were crossbred with either CysC transgenic mice or with CysCKO mice to study the effect of levels of CysC expression on the pathologies observed in the CysB deficient mice.

Performance of mice on the rotorod, a device that allows quantification of motor coordination and balance, was studied to evaluate the effect of the level of CysC expression on deficit in coordination and balance. Mild loss in balance was observed as early as 2 months of age in CysBKO mice, and this characteristic loss increased with age at 4 and 12 months in all of the CysB deficient groups. In contrast, WT, CysC overexpressing, and 

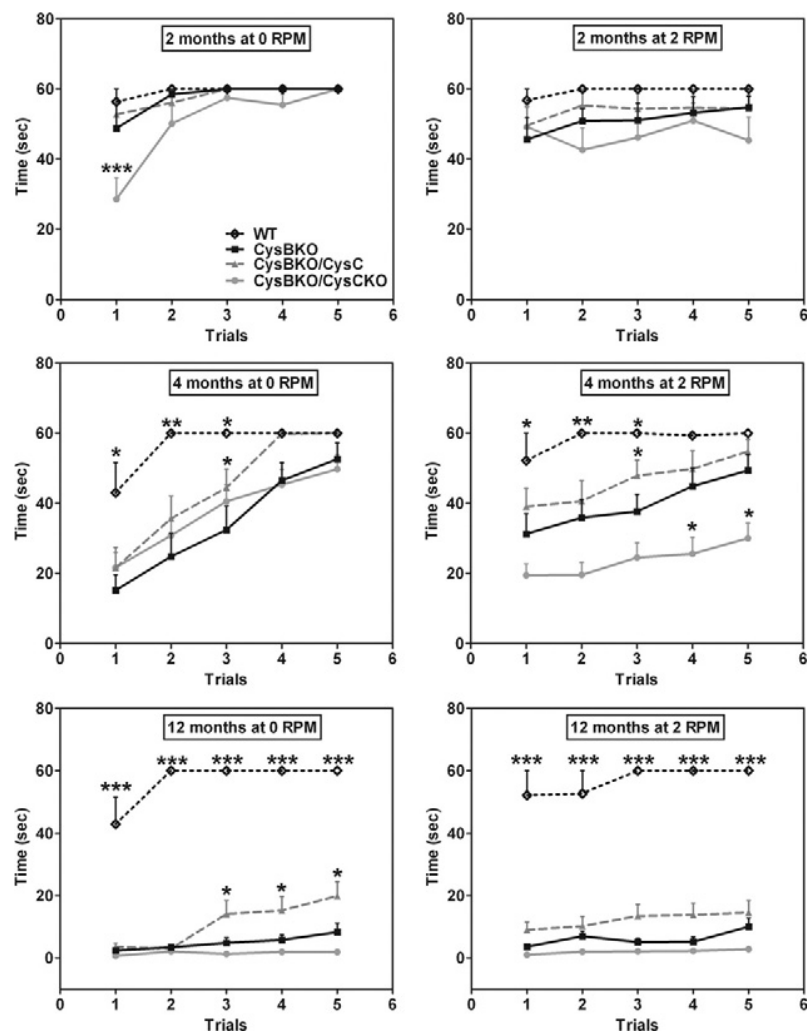

Figure 1. CysC improves performance on rotorod, a measure of motor coordination and balance, in CysBKO mice. Still $(0 \mathrm{rpm})$ and rotating $(2 \mathrm{rpm})$ rods were used to measure motor coordination at 2, 4, and 12 months of age, and the average time spent on the rod was recorded. Mild signs of discoordination, observed in animals as young as 2 months, showed an increase with age in the three CysBKO groups. The overexpression of CysC in CysBKO mice led to less abnormal behavior, whereas CysC deficiency in CysBKO mice minimized the time spent on the rod even further. Measurements are presented as mean \pm SEM (for WT, CysBKO, CysBKO/CysC, and CysBKO/CysCKO, $n=4,11,11$, and 9 at 2 months; $n=4,14,14$, and 23 at 4 months; and $n=4,20,21$, and 6 at 12 months). The differences from CysBKO were significant at ${ }^{*} P<0.05,{ }^{* *} P<0.01$, and ${ }^{* * * *} P<0.001$.

CysCKO mice did not display motor deficits on the rotorod at all age groups (Figure 1). At 2 months of age, mice of all genotypes performed well on a still rod, except for the CysBKO/CysCKO group, which showed a significant decrease in the time they stayed on the rod as compared with CysBKO mice (trial 1; $P<0.001$; Figure 1). At 2 months, all of the three groups of $C y s B$ deficient mice (CysBKO, CysBKO/CysC, and CysBKO/CysCKO mice) showed a trend toward losing the coordination on the rotating rod with a decreased stay on the rod. At 4 months of age, on both a still rod and a rotating rod, CysBKO showed significantly reduced motor coordination as compared with WT mice in trials 1,2 , and $3(P<$
0.05, $P<0.01$, and $P<0.05$, respectively; Figure 1). Overexpression of CysC in CysBKO/CysC mice led to significantly better performance with mice staying longer on the still rod (trial 3; $P<0.05$ ). CysBKO/CysC mice also managed better coordination on the rotating rod (trial 3; $P<0.05$ ), and CysC depletion in CysBKO/CysCKO mice resulted in a significantly worse motor coordination on the rotating rod (trials 4 and $5 ; P<0.05$ ). At 12 months, the three CysB deficient mouse groups performed very poorly with increased imbalance. CysBKO mice were significantly different from WT mice in maintaining the equilibrium on both the still and rotating rods (Figure 1). CysBKO/CysC mice exhibited increased loss of balance with age but could stay on the rod for longer time durations as compared with CysBKO mice on the still and rotating rods ( 0 rpm; trials 3,4 , and $5 ; P<0.05$ each, respectively).

\section{Effect of CysC Overexpression on Brain Atrophy in CysBKO Mice}

Measurement of total body weight and whole brain weight at 12 months of age revealed a statistically significant reduction in CysBKO mice as compared with WT control mice without a difference in brain- to body-weight ratios (Table 1) as observed previously. ${ }^{28}$ CysC overexpression in CysBKO/CysC mice led to increased body weights and brain weights, whereas a drastic reduction in CysBKO/CysCKO animals was observed as compared with CysBKO mice (Table 1). Macroanatomical study of the brains revealed a reduction in brain size and atrophy of the cerebellum and neocortical areas of CysBKO mice as compared with WT mice. Although CysC overexpression in these mice prevented this reduction in the size of the cerebellum, CysC deficiency in CysBKO mice resulted in an additional decrease in the size of the cerebellum. Quantitative analysis of cerebellar atrophy was performed by measuring the size of cerebellar folia on Nissl stained sections (Figure 2, A and B). Cerebellar atrophy was observed at 2 months of age in CysBKO mice and increased with age as compared with WT mice $(P<0.001$; Figure 2C). The effect of CysC depletion in CysBKO/CysCKO mice was evident starting at 2 months of age as the cerebella were more atrophied than seen in CysBKO mice $(P<0.01)$. Cerebellar atrophy was attenuated in CysBKO/CysC mice at 4 and 12 months of age, but was increased in CysBKO/CysCKO mice at these time points (Figure 2C).

Table 1. CysC Partially Rescues Brain and Body Weight Loss in CysBKO Mice

\begin{tabular}{lcccc}
\hline & $\begin{array}{c}\text { Wild type } \\
(n=8)\end{array}$ & $\begin{array}{c}\text { CysBKO } \\
(n=11)\end{array}$ & $\begin{array}{c}\text { CysBKO/CysC } \\
(n=6)\end{array}$ & $\begin{array}{c}\text { CysBKO/CysCKO } \\
(n=6)\end{array}$ \\
\hline Brain weight, g & $0.43 \pm 0.03^{* * *}$ & $0.32 \pm 0.03$ & $0.36 \pm 0.02$ & $0.26 \pm 0.02^{* *}$ \\
Body weight, g & $36.1 \pm 3.89^{* *}$ & $31.0 \pm 3.43$ & $34.5 \pm 2.71$ & $24.3 \pm 0.95^{\star *}$ \\
Brain/body ratio & $0.01 \pm 0.00$ & $0.01 \pm 0.00$ & $0.01 \pm 0.00$ & $0.01 \pm 0.00$ \\
\hline
\end{tabular}

Mean \pm SD of brain weight and body weight in grams and the ratio between the two weights. The differences from CysBKO were significant at ${ }^{* *} P<0.01$ and ${ }^{* *} P<0.001$. 

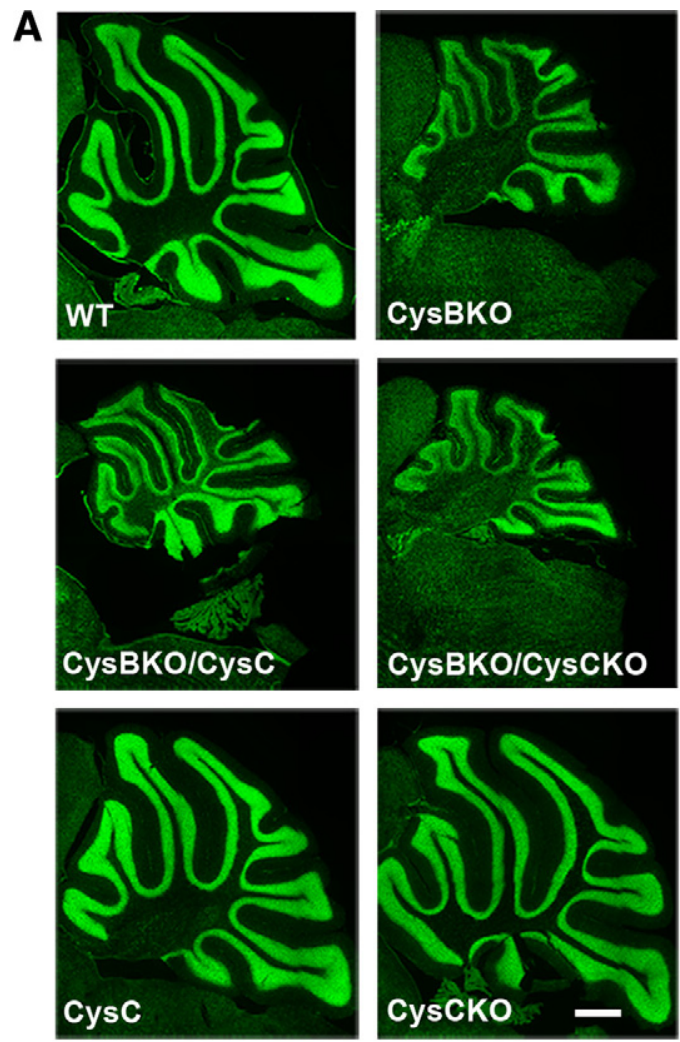

B
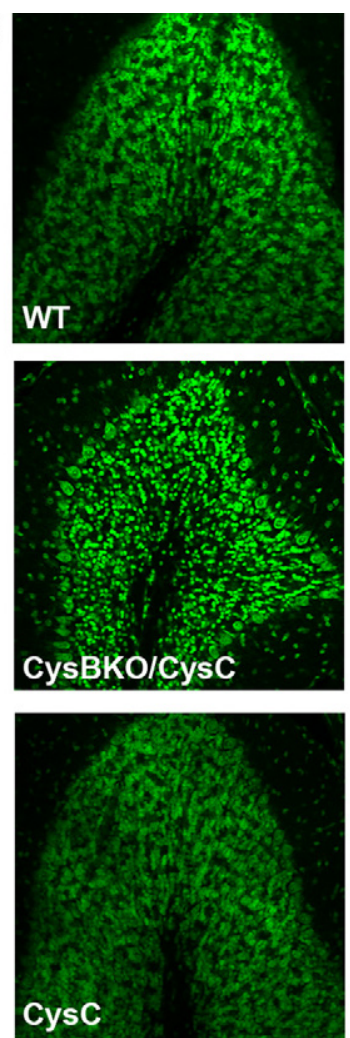
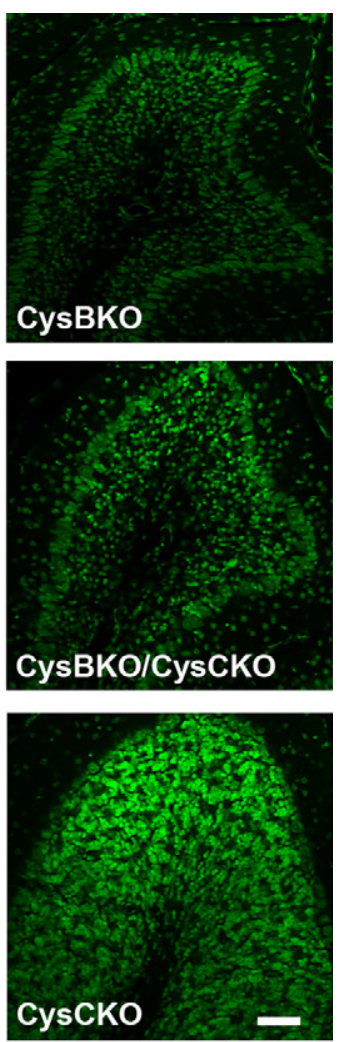

C

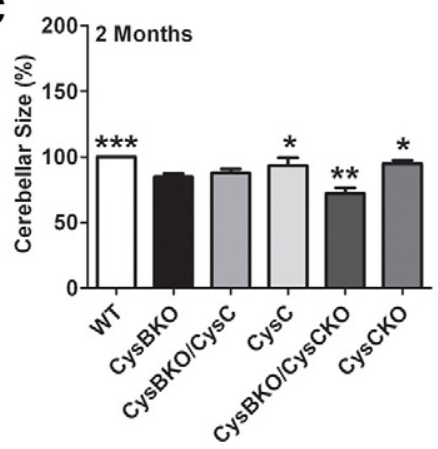

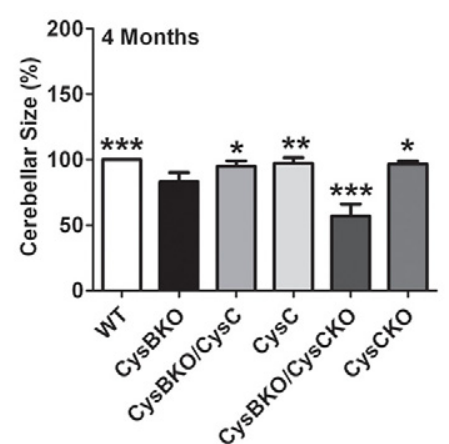

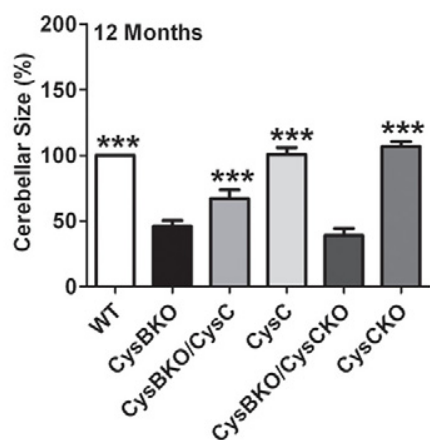

Figure 2. Cerebellar atrophy in CysBKO mice is reversed by CysC overexpression. CysBKO mice showed atrophy and reduced cell density in the granular layer of the cerebellum with increasing age. A and B: Nissl-stained sagittal sections in 12-month-old mice. Scale bars, $500 \mu \mathrm{m}(\mathbf{A})$ and $50 \mu \mathrm{m}(\mathbf{B})$. C: Reduction in the cerebellar size by CysB deficiency in mice at 2, 4, and 12 months of age. Measurement of the area of cerebellar folia revealed that CysC overexpression was protective against CysBKO-induced atrophy. Measurements are presented as mean $\pm \mathrm{SD}(n=4 / 5)$. The differences from CysBKO were significant at ${ }^{*}<<0.05$, ${ }^{* * *} P<0.01$, and ${ }^{* * * *} P<0.001$.

\section{CysC Overexpression Inhibits the Cell Loss Caused by CysB Deficiency}

In addition to cerebellar atrophy, ${ }^{39}$ neuronal degeneration has been previously shown in the cortical motor areas of the brains of patients with EPM1, in correlation with motor symptoms of the disease. ${ }^{40}$ Immunocytochemical staining with anti-NeuN antibody revealed a loss of NeuN immunoreactivity with increasing age in the brains of CysBKO mice starting at 2 months of age. Neuronal loss was most pronounced in the cerebellar granular layer of CysBKO mice when compared with WT (Figure 3A). Western blot analysis of cerebellar homogenates of 4 months old mice with the anti-NeuN antibody revealed two bands of 46 and $48 \mathrm{kDa}$ (Figure 3B), which were quantified and presented as a ratio of the $\beta$-actin band in the same lane for each subject. Results indicate a significant neuronal loss in CysBKO mice as compared with WT mice $(P<0.001)$. Importantly, CysC overexpression in CysBKO/CysC mice partially rescued the neuronal loss phenotype as compared with CysBKO mice $(P<$ 0.05; Figure 3C).

Gliosis Induced by CysB Deficiency Is Reduced by CysC Overexpression

Immunostaining for GFAP revealed a profound gliosis in the brains of 4-month-old CysBKO mice, staining white matter tracts, and glial cells in the subicular complex, 
A

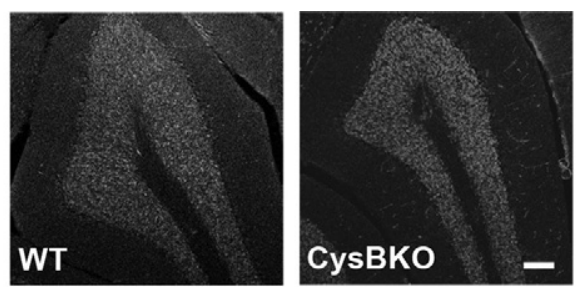

B

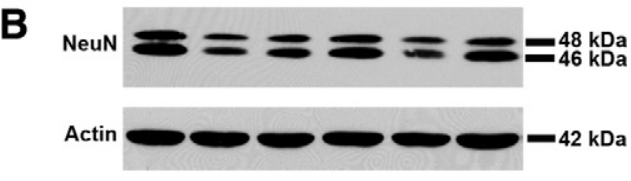

C

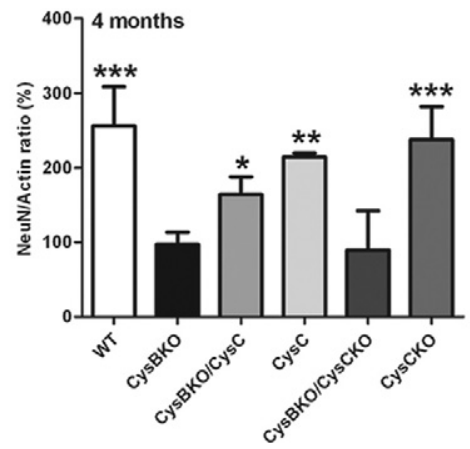

Figure 3. CysC protects against neuronal loss in the cerebella of CysBKO mice. A: Cerebellar sections from 4-month-old mice immunostained with an anti-NeuN antibody show granule cell layer loss in the cerebella of CysBKO compared with WT mice. Scale bar, $100 \mu \mathrm{m}$. B: Western blot analysis of cerebellar homogenates with anti-NeuN antibody shows increased level of expression in CysBKO/CysC mice compared with CysBKO mice. C: Quantification of the ratio of NeuN to $\beta$-actin bands are presented as the mean \pm $\mathrm{SD}(n=4 / 5)$. The differences from CysBKO were significant at ${ }^{*} P<0.05$, ${ }^{* *} P<0.01$, and ${ }^{* * * *} P<0.001$

neocortex, hippocampus, and cerebellum (Figure 4A). Real time qPCR was used to test the effect of CysB deletion on GFAP mRNA levels, showing a significant increase in CysBKO PCR products in the cerebral cortex and cerebellum as compared with WT mice (Figure 4B). Western blot analysis in cortical tissue homogenates demonstrated a concomitant robust increase in GFAP protein expression in CysBKO mice as compared with WT mice at 4 months of age (Figure 4). Similar observations were found at 2 and 12 months of age (data not shown). In contrast, decrease in GFAP expression was observed in CysBKO/CysC mice compared with CysBKO mice, whereas profound gliosis was observed in Cys$\mathrm{BKO} / \mathrm{CysCKO}$ mice (Figure 4C). CysC and CysCKO mice did not show any significant change in GFAP immunoreactivity as compared with WT mice. Immunocytochemical analysis confirmed that the intensity of gliosis was reduced in CysC overexpressing CysBKO/CysC mice, whereas CysBKO/CysCKO mice showed an increase in GFAP immunoreactivity (Figure 4D).

\section{Localization of CysB Expression in the Brains of Wild Type Mice}

We used an affinity purified polyclonal anti-rat CysB antibody that detects by Western blot analysis a protein of $12 \mathrm{kDa}$ in the cerebellum of WT, CysC transgenic, and CysCKO mice, but not in the brains of CysBKO mice
(Figure 5A). Comparison of the intensity of the CysBlabeled bands demonstrated that $\mathrm{Cys} C$ transgenic and CysCKO mice express similar levels of CysB as compared with WT mice, indicating no enhanced expression of CysB to compensate for the loss of CysC in the brains of CysCKO mice (Figure 5A). Immunocytochemistry revealed greater CysB staining intensity in the cerebellum compared with the cerebral cortex. CysB immunoreactivity was the strongest in Purkinje cells and in Bergmann glia, exhibiting predominantly punctate staining patterns within the cytoplasm as well as a diffuse distribution throughout the cytoplasm (Figure 5B). Double immunostaining with antibodies directed against CysB and the lysosomal marker LAMP-2 confirmed the cellular colocalization (Figure 5, C and D). These immunocytochemical data suggest that CysB is localized within the cytoplasm and lysosomal compartments.

\section{Compensation for CysB Deficiency by Increased CysC Expression in the Brains of CysBKO Mice}

Increased CysC immunoreactivity was observed in the cerebral cortex and cerebellum of CysBKO mice compared with WT controls by immunocytochemistry (Figure 6, $A$ and $B$ ) and by Western blot analysis (Figure 6, C-F). No CysC expression was observed in CysCKO and CysBKO/CysCKO brains (Figure 6, C and E). Quantification of the 14-kDa CysC band revealed an increase in CysC protein levels in the cortices and cerebella of CysBKO mice compared with WT animals $(P<0.05$; Figure 6, $\mathrm{C}-\mathrm{F}$ ), and CysBKO/CysC compared with CysC mice (Figure $6, \mathrm{C}-\mathrm{F})$. Similar results were obtained at 2 and 12 months of age in the cerebral cortex and cerebellum (data not shown).

\section{Deletion of CysB Enhances mRNA Levels of the Proteases Cat $B$ and Cat $D$ and the Protease Inhibitor CysC}

Real time qPCR was used to test the effect of CysB deletion on the mRNA levels of CysB, CysC, Cat B, Cat D, and GAPDH in CysBKO and WT mice. Analysis of CysB mRNA levels in cerebral cortex and cerebellum verified the CysB gene deletion in CysBKO mice (data not shown). Quantitative analyses showed that CysC mRNA levels were significantly increased in CysBKO mice in both cerebral cortex $(P<0.001)$ and cerebellum $(P<$ 0.03 ) in comparison with WT mice at 9 to 10 months of age (Figure 7A). CysCKO mice, analyzed as controls, did not show any CysC mRNA expression, and CysB mRNA levels in CysCKO mice were similar to WT mice (data not shown). Although a trend of increased Cat B mRNA expression was found in CysBKO mice, the difference compared with WT did not attain significance (Figure 7B). Cat D mRNA was significantly elevated in cortices $(P<0.01)$ and cerebella $(P<0.03)$ of CysBKO mice compared with WT mice (Figure 7C). 
A

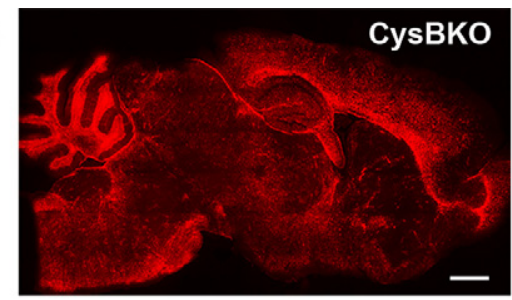

B

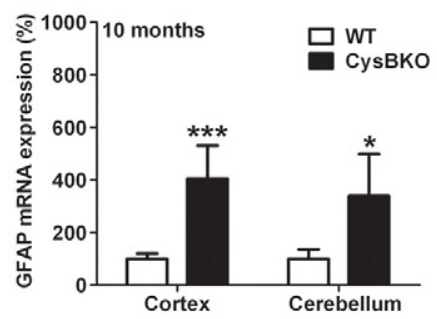

C

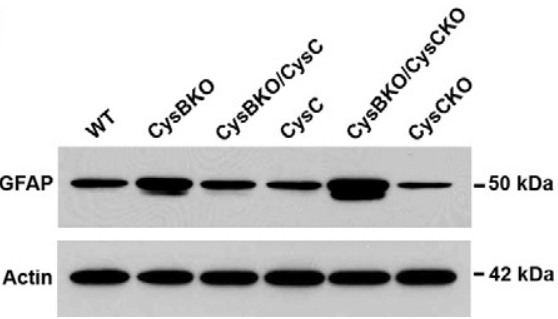

D
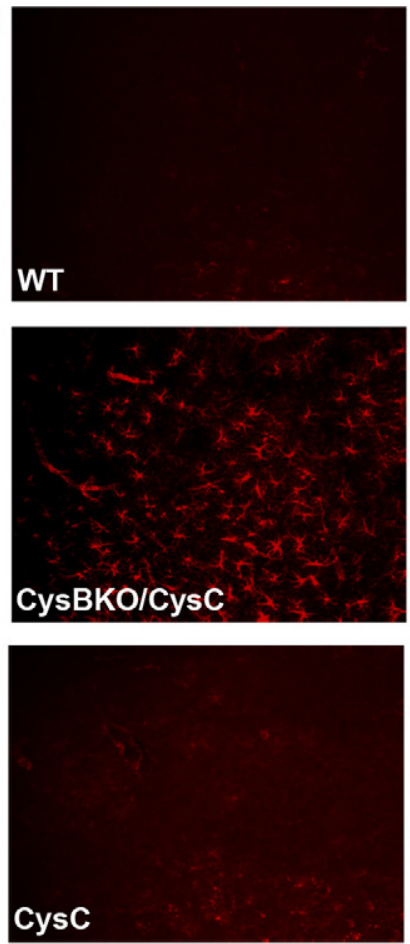
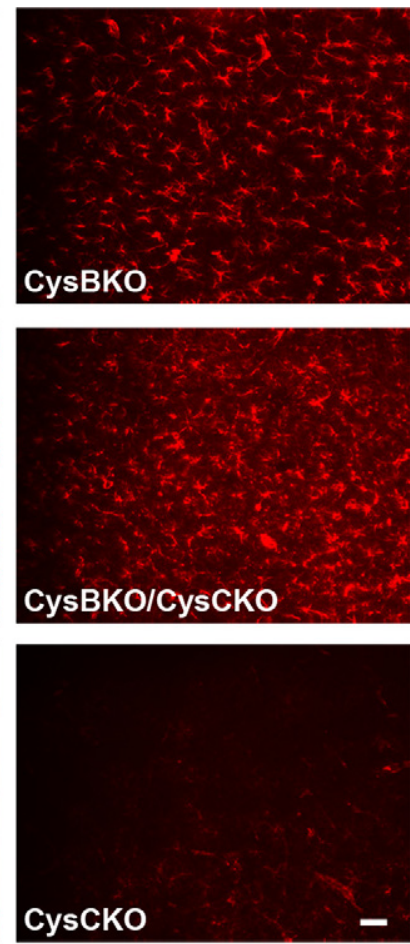

Figure 4. Gliosis induced by CysB deletion is attenuated by CysC overexpression. A: Immunocytochemical staining with anti-GFAP antibody of a sagittal section from a CysBKO mouse revealed intense astrogliosis in gray and white matter areas of both the cerebrum and cerebellum (Scale bar, 1 mm). B: CysB deficiency induces GFAP mRNA expression. qPCR analysis of GFAP is presented relative to mRNA expression of GAPDH in the cortices and cerebella of CysBKO and WT mice. Measurements are presented as the mean $\pm \mathrm{SD}(n=4 / 5)$. The differences from WT were significant at ${ }^{*} P<0.05$ and ${ }^{* * * *} P<0.001$. C: Western blot analysis of brain cortical homogenates with anti-GFAP antibody confirms the effect of CysC level of expression on gliosis in CysBKO mice. D: Immunostaining of cerebral cortex of 4-month-old mice showed that overexpression of CysC in CysBKO mice reduces GFAP staining, whereas CysC deficiency in CysBKO mice exacerbated the pathology (Scale bar, $100 \mu \mathrm{m})$.

\section{CysC Reverses the Impact of CysB Deletion on the Levels of Expression and Activity of Lysosomal Enzymes}

CysB deficiency has been shown previously to increase cathepsin activity. ${ }^{16}$ Accordingly, we found that Cat B and Cat D immunostaining was increased in the brains of CysBKO mice as compared with WT mice, although deletion of $\mathrm{CysB}$ had a stronger effect on Cat $D$ expression levels compared with Cat B (Figures 8A and 9A). Bio- chemical analyses of Cat B and Cat D activities were performed in cortical and cerebellar homogenates of 4-month-old mice. CysBKO mice showed an increase in Cat B activity as compared with WT mice in both cerebral cortex and cerebellum ( $P<0.05$; Figure 8 , B and $\mathrm{C}$ ). A trend of decrease in Cat B activity was observed in CysBKO/CysC mice as compared with CysBKO mice, and knocking out both $\mathrm{CysB}$ and $\mathrm{Cys} C$ led to a significant increase in Cat $\mathrm{B}$ activity in CysBKO/CysCKO mice when compared with CysBKO mice $(P<0.05)$ in both cerebral
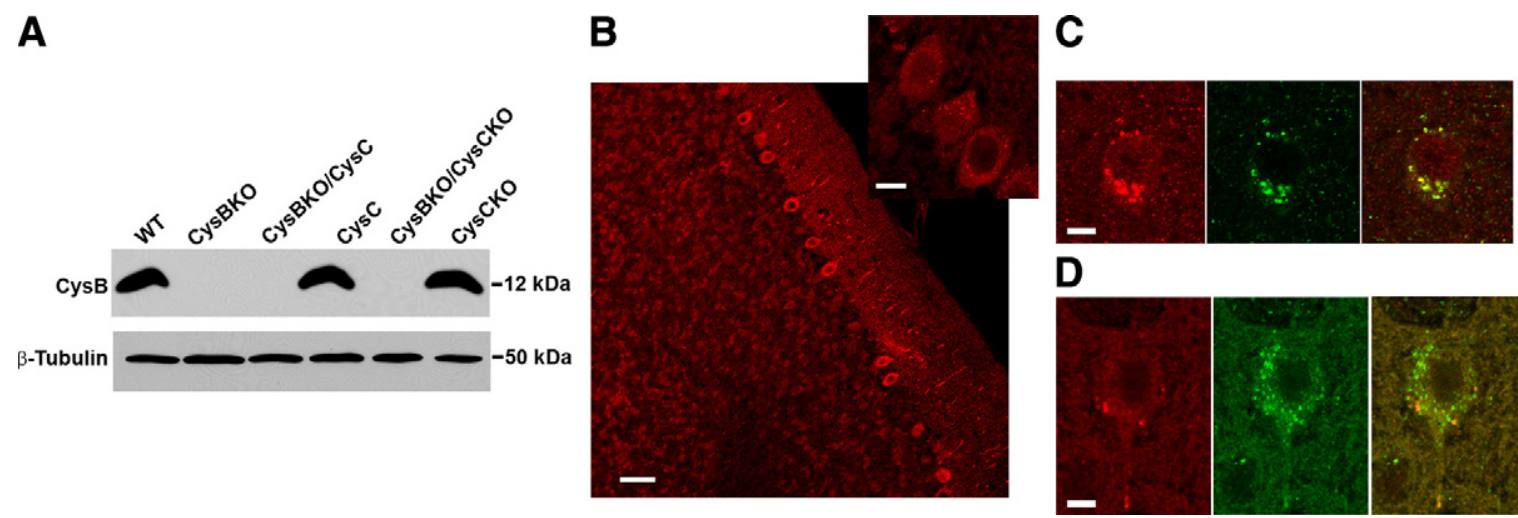

Figure 5. Localization of CysB in cerebellar neurons. A: Western blot analysis with anti-CysB antibody of cerebellar homogenates of 4-month-old mice shows no immunoreactivity in the brains of CysBKO mice. B: Immunofluorescent labeling of CysB in the cerebellum of a WT mouse shows localization mainly in Purkinje cells and Bergmann's glial cells in the molecular layer. Both cytosolic and punctate staining was observed in Purkinje cells. Inset shows the CysB punctate staining within Purkinje cells (Scale bars, $100 \mu \mathrm{m}$ and $10 \mu \mathrm{m}$ [inset]). C and D: Double-immunolabeling with antibodies to CysB (red) and to LAMP-2 (green) show colocalization in cortical neurons, suggesting lysosomal expression of CysB (Scale bar, $10 \mu \mathrm{m}$ ). 

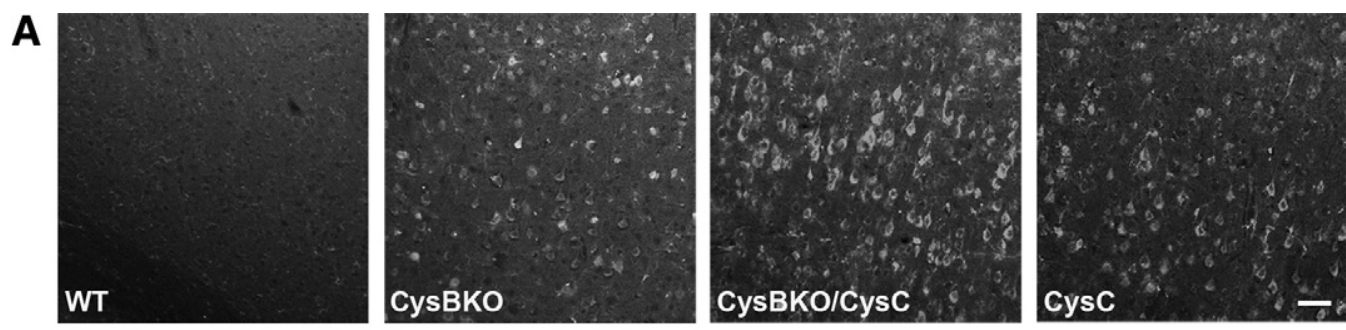

B
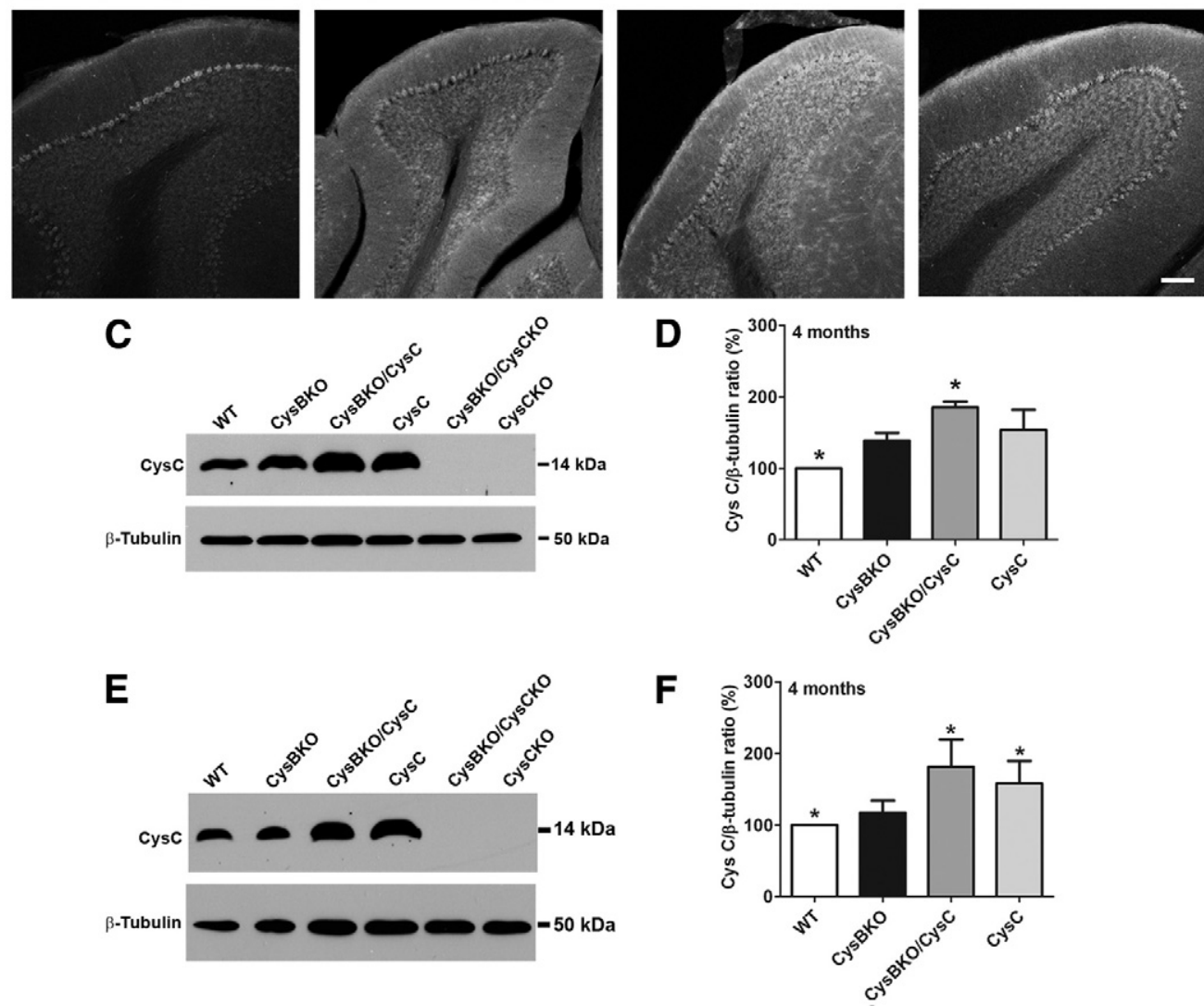

Figure 6. Enhanced CysC expression levels in the brains of CysBKO mice. Brain sections immunostained with anti-CysC antibody showed increased expression levels of CysC in CysBKO mice compared with WT mice in the cerebral cortex (A; Scale bar, $50 \mu \mathrm{m})$ and cerebellum (B; Scale bar, $100 \mu \mathrm{m})$. C and $\mathbf{E}$ : Western blot analysis of cerebellar and cortical homogenates, respectively, with anti-CysC antibody confirms an increase in CysC expression levels in CysBKO mice compared with WT mice and in CysBKO/CysC mice compared with CysC singly transgenic mice. D and F: Quantification of the ratio of CysC to $\beta$-tubulin bands in cerebellum and cerebral cortex, respectively, presented as the mean $\pm \mathrm{SD}(n=4 / 5)$. The differences from CysBKO were significant at ${ }^{*} P<0.05$.

cortex and cerebellum (Figure 8, B and C). Cat D was significantly increased in the cortices $(P<0.01)$ and cerebella $(P<0.001)$ of CysBKO mice compared with WT mice (Figure 9, B and C). Although Cat D activity was decreased in the cortices of CysBKO/CysC mice compared with CysBKO mice $(P<0.05)$, CysC depletion in CysBKO/CysCKO mice did not affect Cat D activity when compared with CysBKO mice (Figure 9, B and C).

\section{Discussion}

CysB Deficiency Leads to Lysosomal Pathology in Brain Tissues

CysB gene mutation resulting in loss-of-function is responsible for the severe neurological disorder, EPM1. Cerebellar atrophy and motor cortex degeneration has
A

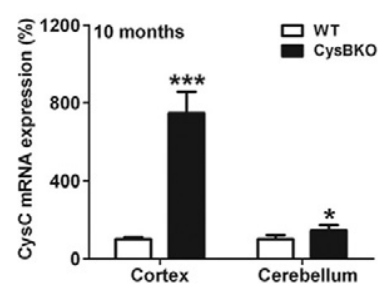

\section{B}

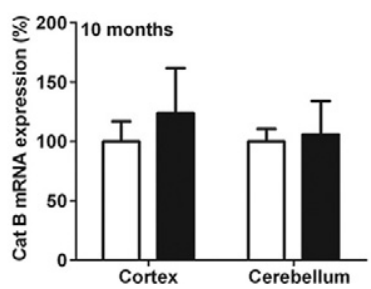

\section{C}

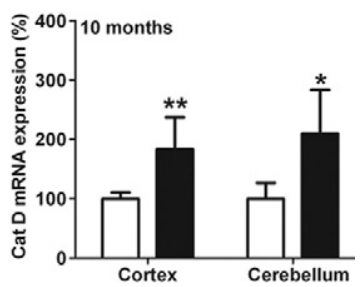

Figure 7. CysB deficiency increases mRNA levels of Cat B, Cat D, and CysC. qPCR analysis of CysC (A), Cat B (B), and Cat D $(\mathbf{C})$ is depicted relative to mRNA expression of GAPDH in the cortices and cerebella of CysBKO and WT mice. Measurements are presented as the mean \pm SD $(n=4 / 5)$. The differences from WT were significant at ${ }^{*} P<0.05,{ }^{* *} P<0.01$, and ${ }^{* * * *} P<0.001$. 

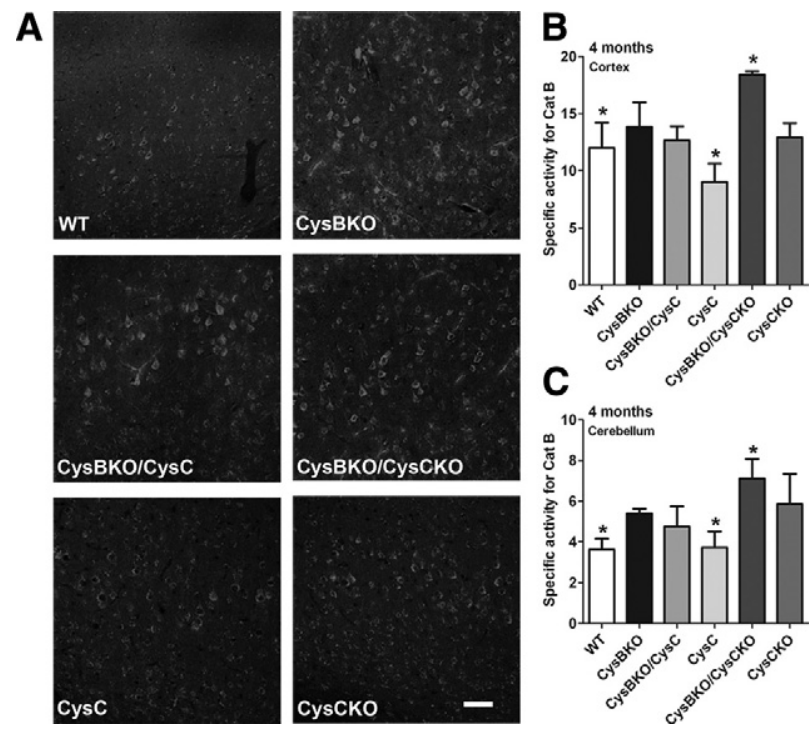

Figure 8. Increased Cat B immunoreactivity and activity in CysBKO mice. A: Immunocytochemical staining with anti-Cat B antibody of the cerebral cortex of mice at 4 months of age (Scale bar, $50 \mu \mathrm{m}$ ). B and C: Proteolytic activity of Cat B in cortical (B) and cerebellar (C) homogenates expressed as nanomole per minute per milligram of protein. Measurements are presented as the mean $\pm \mathrm{SD}(n=4 / 5)$. The differences from CysBKO were significant at ${ }^{*} P<0.05$.

been shown to occur in patients with EPM1, correlating with the motor symptoms of the disease. ${ }^{39,40}$ Similar to patients with EPM1, CysBKO mice display myoclonic seizures, progressive ataxia, loss of motor coordination, and cerebellar pathology. The principal cytopathology of CysBKO mice appears to be a loss of cerebellar granule cells, ${ }^{27}$ mimicking the changes seen in patients with EPM1. Apoptotic neuronal loss was also described within the hippocampal formation and entorhinal cortex at 3 to 4
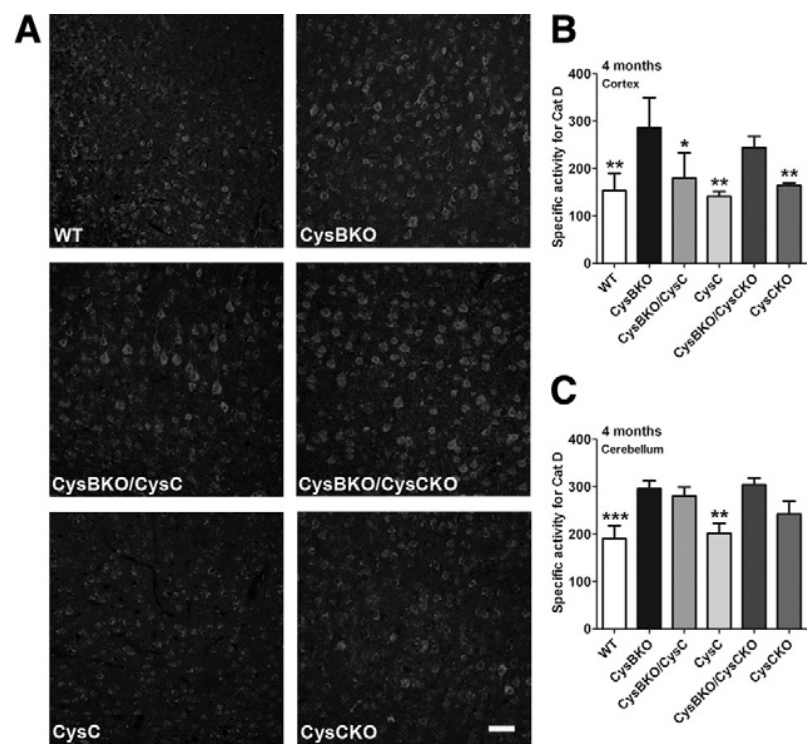

C

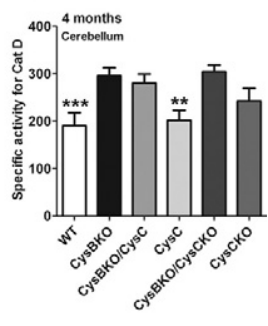

Figure 9. Cat D immunoreactivity and activity in CysBKO mice. A: Immunocytochemical staining with anti-Cat D antibody of the cerebral cortex of 4-month-old mice (Scale bar, $50 \mu \mathrm{m}$ ). B and C: Proteolytic activity of Cat D in cortical (B) and cerebellar $(\mathbf{C})$ homogenates expressed as nanomole per minute per milligram of protein. Measurements are presented as the mean \pm $\mathrm{SD}(n=4 / 5)$. The differences from CysBKO were significant at ${ }^{*} P<0.05$ ${ }^{* * *} P<0.01$, and ${ }^{* * * * *} P<0.001$ months of age, and in older mutant mice (16 to 18 months old), accompanied by gliosis, most marked in the subicular complex and hippocampus, as well as the entorhinal cortex, neocortex, striatum, and in the white matter. ${ }^{28}$ Using immunohistochemical and Western blot analyses, we confirmed the age-related neuronal loss in the cerebellum of CysBKO mice. Similar to a previous demonstration, ${ }^{17}$ we also show enhanced GFAP mRNA and protein levels, indicative of widespread gliosis in the brains of CysBKO mice. Immunohistochemical staining of the brains of WT mice with an anti-CysB antibody revealed stronger CysB staining in the cerebellum compared with the cerebral cortex, with the strongest expression in Purkinje cells and in Bergmann glia (Figure 5). ${ }^{41}$ This differential distribution of CysB expression may account for the greater vulnerability of cerebellar neurons observed in CysBKO mice.

CysB immunostaining in brains of WT mice in this study shows cytoplasmic and lysosomal localization, supporting earlier findings that CysB is distributed diffusely throughout the cytoplasm ${ }^{42}$ and localized mainly within lysosomal compartments. ${ }^{43}$ We confirm and extend the reports that CysB has a role in lysosomal functioning and that loss-of-function in EPM1 and subsequent lysosomal dysfunction are the major pathogenic factors in the disease. ${ }^{43}$

Increased cathepsins' enzymatic activity has been associated with the loss of CysB. ${ }^{16}$ Earlier studies hypothesized that augmented proteolysis by lysosomal cathepsins, mainly by $\mathrm{Cat} \mathrm{B}$, is responsible for phenotypic characteristics of EPM1 and deletion of Cat B in CysBKO mice resulted in a reduction in the amount of cerebellar granule cell apoptosis depending on mouse age. ${ }^{15}$ This finding established Cat $\mathrm{B}$ as a contributor to the apoptotic phenotype of CysBKO mice and humans with EPM $1 .{ }^{15}$ Herein, we also demonstrate increased mRNA, protein, and enzymatic activity levels of the two lysosomal enzymes Cat B and Cat D in the brains of CysBKO mice. Primary lysosomal dysfunction in lysosomal storage disorders was suggested as the cause of severe neurodegenerative phenotypes. ${ }^{44}$ Increased cathepsin activities and apoptotic nuclear changes in cerebellar granule cells suggest that CysB deficiency causes lysosomal dysfunction and subsequent oxidative damage resulting in neurodegeneration and cell death.

\section{CysC Prevents Neurodegeneration Caused by CysB Deficiency}

We tested the hypothesis that CysC overexpression can rescue the loss of function of CysB in CysBKO mice. We observed an endogenous compensatory up-regulation of CysC in CysBKO mice, with a sevenfold increase in CysC mRNA levels and increased CysC protein expression levels in the brains of CysBKO mice as compared with WT mice. The compensatory activation of CysC occurs despite the different subcellular localization of the two inhibitors. Although CysB is intracellular, with mainly lysosomal and cytoplasmic localization, the primary struc- 
ture of CysC is indicative of a secreted protein and accordingly, it has been demonstrated that most of the CysC is targeted extracellularly via the secretory pathway (reviewed in Levy et al). ${ }^{45}$ Enhanced CysC expression occurs in human patients with epilepsy, in animal models of neurodegenerative conditions, and in response to injury, including facial nerve axotomy, noxious input to the sensory spinal cord, perforant path transections, hypophysectomy, and transient forebrain ischemia (reviewed in Levy et al). ${ }^{45}$ Thus, an increase in CysC expression in CysBKO mice might represent an intrinsic neuroprotective mechanism to rescue neurons by inhibiting the apoptosis-promoting actions of cathepsins. However, the level of CysC expression in these mice may not be sufficient to counteract the progression of the disease. The effect of CysC overexpression was observed on motor coordination and balance as tested by performance on rotorod, cerebellar atrophy, neuronal loss in the cerebellum, and gliosis, revealing that CysC partially compensates for loss of cysteine protease inhibition in the absence of CysB.

In vitro experiments have indicated that both $\mathrm{CysB}$ and CysC can inhibit cathepsins $\mathrm{B}, \mathrm{H}, \mathrm{L}, \mathrm{S}$, and $\mathrm{K} .{ }^{46}$ We found increased Cat $B$ and Cat D mRNA levels and enzymatic activities in the brains of CysBKO mice. These findings are consistent with those in cells from patients with EPM1 in which decreased levels of CysB mRNA and reduced CysB protein levels correlated with increased activities of $\mathrm{Cat} B, \mathrm{Cat} \mathrm{L}$, and Cat $\mathrm{S} .{ }^{16}$ CysC deficiency in CysBKO/CysCKO mice further elevated cathepsin activity as compared with CysBKO mice, leading to aggravated pathologies. Although we show that $\mathrm{Cys} C$ overexpression in Cys $\mathrm{C}$ transgenic mice did not affect the enzymatic activity of Cat B and Cat D, CysC overexpression in CysBKO mice decreases Cat $\mathrm{B}$ and $\mathrm{Cat} \mathrm{D}$ activities in the brains of the CysBKO/ CysC mice.

These data show that CysC overexpression partially prevents neurodegeneration in CysBKO mice through inhibition of cathepsins activity. Although CysC can inhibit cysteine proteases, protease-independent activities have been shown previously. ${ }^{47-49}$ We have observed that in neuronal cultures exposed to cytotoxic challenges, the neuroprotective action of $\mathrm{Cys} C$ does not require $\mathrm{Cat}$ $\mathrm{B}$ inhibition ${ }^{25}$ but involves induction of fully functional autophagy via the mTOR pathway, leading to proteolytic clearance of autophagy substrates by lysosomes. ${ }^{25}$ Moreover, CysC inhibits $A \beta$ oligomerization ${ }^{50}$ and fibril formation in vitro ${ }^{51}$ and in vivo. ${ }^{33,52}$ CysC also increases cell survival when added together with preformed oligomeric or fibrillar $A \beta$ to either cultured primary hippocampal neurons or to N2a cells. ${ }^{26}$ Although CysC inhibits $A \beta$ aggregation, it does not disrupt preformed $A \beta$ fibrils or oligomers. ${ }^{50,51}$ Thus, CysC exhibits a dual protective effect against $A \beta$ toxicity, inhibiting $A \beta$ aggregation in addition to the direct neuroprotective effect that is independent of its anti-A $\beta$ amyloidogenic property, dependent on pathways such as induction of autophagy and inhibition of cysteine proteases.

\section{CysC Overexpression Reverses the Pathophysiology Induced by CysB Deficiency}

CysC level of expression in CysBKO mice has multiple effects on the pathologies observed in these mice. CysC overexpression in CysBKO mice decreased cerebellar granule cell loss, reduced gliosis, and as a result, improved motor coordination and reduced the extent of imbalance. The loss of CysC in CysBKO/CysCKO mice had an opposing deteriorating effect. There are several possible explanations for the fact that none of the pathologies studied recovered to WT control levels by overexpression of CysC in CysBKO mice in this study. We cannot rule out the possibility that higher CysC levels have deleterious effects on cell viability. CysC overexpression in $\mathrm{CysBKO} / \mathrm{Cys} \mathrm{C}$ mice above the elevated CysC levels in CysBKO mice may lead to localized toxic levels of the protein, similar to the observation that CysC injection into rat hippocampus caused neuronal degeneration in the site of the injection. ${ }^{53}$ Alternatively, it is possible that CysC cannot compensate for all CysB functions, due to different cellular localization and/or inhibitory activities. Although CysC is a secreted protein and is internalized into cells, ${ }^{54}$ CysB is localized mainly to lysosomes and its deletion impairs lysosomal functioning. ${ }^{30,43}$ Moreover, although CysB is highly expressed in Purkinje cells and Bergmann glia, ${ }^{15,27,28}$ Cys C levels of expression in these cells are comparable to other regions of the brain. Finally, the level of Cys $\mathrm{C}$ expression in the crossed mice could be insufficient to counteract the full lysosomal dysfunction due to loss of CysB.

Anti-epileptic drug treatments are used currently as a therapeutic intervention in patients with EPM1. ${ }^{55}$ Longterm side effects of these drugs can be avoided if other treatment modalities are developed and implemented. Our findings showing that CysC plays a protective role under conditions of neuronal challenge are consistent with Cys $\mathrm{C}$ being neuroprotective in neurodegenerative diseases such as EPM1. We demonstrate here that CysC, or a CysCmimicking analog, could be a therapeutic candidate with a potential of preventing neurodegeneration. Also, this work has strong implications for Alzheimer's disease and other age-related neurodegenerative disorders where lysosomal dysfunction plays a considerable role. ${ }^{44}$

\section{Acknowledgments}

We are grateful to Drs. Goutam Chakraborty and Appa Hungund for their assistance, and Dr. Richard M. Myers for providing a breeding colony of CysBKO mice.

\section{References}

1. Pennacchio LA, Lehesjoki AE, Stone NE, Willour VL, Virtaneva K, Miao J, D'Amato E, Ramirez L, Faham M, Koskiniemi M, Warrington JA, Norio R, de la Chapelle A, Cox DR, Myers RM: Mutations in the gene encoding cystatin B in progressive myoclonus epilepsy (EPM1). Science 1996, 271:1731-1734

2. Lalioti MD, Scott HS, Buresi C, Rossier C, Bottani A, Morris MA Malafosse A, Antonarakis SE: Dodecamer repeat expansion in cys- 
tatin B gene in progressive myoclonus epilepsy. Nature 1997, 386:847-851

3. Lafreniere RG, Rochefort DL, Chretien N, Rommens JM, Cochius JI, Kalviainen R, Nousiainen U, Patry G, Farrell K, Soderfeldt B, Federico A, Hale BR, Cossio OH, Sorensen T, Pouliot MA, Kmiec T, Uldall P, Janszky J, Pranzatelli MR, Andermann F, Andermann E, Rouleau GA: Unstable insertion in the $5^{\prime}$ flanking region of the cystatin $B$ gene is the most common mutation in progressive myoclonus epilepsy type 1 , EPM1. Nat Genet 1997, 15:298-302

4. Koskiniemi M, Donner M, Majuri H, Haltia M, Norio R: Progressive myoclonus epilepsy: a clinical and histopathological study. Acta Neurol Scand 1974, 50:307-332

5. Norio R, Koskiniemi M: Progressive myoclonus epilepsy: genetic and nosological aspects with special reference to 107 Finnish patients. Clin Genet 1979, 15:382-398

6. Eldridge R, livanainen M, Stern R, Koerber T, Wilder BJ: "Baltic" myoclonus epilepsy: hereditary disorder of childhood made worse by phenytoin. Lancet 1983, 2:838-842

7. Franceschetti S, Sancini G, Buzzi A, Zucchini S, Paradiso B, Magnaghi G, Frassoni C, Chikhladze M, Avanzini G, Simonato M: A pathogenetic hypothesis of Unverricht-Lundborg disease onset and progression. Neurobiol Dis 2007, 25:675-685

8. Barrett AJ: The cystatins: a diverse superfamily of cysteine peptidase inhibitors. Biomed Biochim Acta 1986, 45:1363-1374

9. Barrett AJ, Fritz H, Grubb A, Isemura S, Jarvinen M, Katunuma N, Machleidt W, Muller-Esterl W, Sasaki M, Turk V: Nomenclature and classification of the proteins homologous with the cysteine-proteinase inhibitor chicken cystatin. Biochem J 1986, 236:312

10. Turk V, Bode W: The cystatins: protein inhibitors of cysteine proteinases. FEBS Lett 1991, 285:213-219

11. Tizon B, Levy E: Protease inhibitors and their involvement in neurological disorders. Edited by A Lajtha. New York, Springer Publishers, 2006, pp 591-624

12. Turk B, Turk D, Turk V: Lysosomal cysteine proteases: more than scavengers. Biochim Biophys Acta 2000, 1477:98-111

13. Kirschke H, Barrett AJ, Rawlings ND: Lysosomal cysteine proteinases. Edited by P Sheterline. London, Academic Press, 1995, pp $1581-1643$

14. Kos J, Werle B, Lah T, Brunner N: Cysteine proteinases and their inhibitors in extracellular fluids: markers for diagnosis and prognosis in cancer. Int J Biol Markers 2000, 15:84-89

15. Houseweart MK, Pennacchio LA, Vilaythong A, Peters C, Noebels JL, Myers RM: Cathepsin B but not cathepsins $L$ or $S$ contributes to the pathogenesis of Unverricht-Lundborg progressive myoclonus epilepsy (EPM1). J Neurobiol 2003, 56:315-327

16. Rinne R, Saukko P, Jarvinen M, Lehesjoki AE: Reduced cystatin B activity correlates with enhanced cathepsin activity in progressive myoclonus epilepsy. Ann Med 2002, 34:380-385

17. Lieuallen K, Pennacchio LA, Park M, Myers RM, Lennon GG: Cystatin B-deficient mice have increased expression of apoptosis and glial activation genes. Hum Mol Genet 2001, 10:1867-1871

18. Ishimaru H, Ishikawa K, Ohe Y, Takahashi A, Maruyama Y: Cystatin C and apolipoprotein $\mathrm{E}$ immunoreactivities in $\mathrm{CA} 1$ neurons in ischemic gerbil hippocampus. Brain Res 1996, 709:155-162

19. Palm DE, Knuckey NW, Primiano MJ, Spangenberger AG, Johanson CE: Cystatin C, a protease inhibitor, in degenerating rat hippocampal neurons following transient forebrain ischemia. Brain Res 1995, 691:1-8

20. Hendriksen H, Datson NA, Ghijsen WE, van Vliet EA, da Silva FH, Gorter JA, Vreugdenhil E: Altered hippocampal gene expression prior to the onset of spontaneous seizures in the rat post-status epilepticus model. Eur J Neurosci 2001, 14:1475-1484

21. Aronica E, van Vliet EA, Hendriksen E, Troost D, Lopes da Silva FH, Gorter JA: Cystatin C, a cysteine protease inhibitor, is persistently up-regulated in neurons and glia in a rat model for mesial temporal lobe epilepsy. Eur J Neurosci 2001, 14:1485-1491

22. Lukasiuk K, Pirttila TJ, Pitkanen A: Upregulation of cystatin C expression in the rat hippocampus during epileptogenesis in the amygdala stimulation model of temporal lobe epilepsy. Epilepsia 2002, 43(Suppl 5):137-145

23. Nishio C, Yoshida K, Nishiyama K, Hatanaka H, Yamada M: Involvement of cystatin $\mathrm{C}$ in oxidative stress-induced apoptosis of cultured rat CNS neurons. Brain Res 2000, 873:252-262

24. Wang Z, Wu D, Vinters HV: Hypoxia and reoxygenation of brain microvascular smooth muscle cells in vitro: cellular responses and expression of cerebral amyloid angiopathy-associated proteins. Apmis 2002, 110:423-434

25. Tizon B, Sahoo S, Yu H, Gauthier S, Kumar AR, Mohan P, Figliola M, Pawlik M, Grubb A, Uchiyama Y, Bandyopadhyay S, Cuervo AM, Nixon RA, Levy E: Induction of autophagy by cystatin C: a mechanism that protects murine primary cortical neurons and neuronal cell lines. PLoS One 2010, 5:e9819

26. Tizon B, Ribe EM, Mi W, Troy CM, Levy E: Cystatin C protects neuronal cells from amyloid $\beta$-induced toxicity. J Alzheimers Dis 2010, 19:665-894

27. Pennacchio LA, Bouley DM, Higgins KM, Scott MP, Noebels JL, Myers RM: Progressive ataxia, myoclonic epilepsy and cerebellar apoptosis in cystatin B-deficient mice. Nat Genet 1998, 20:251-258

28. Shannon P, Pennacchio LA, Houseweart MK, Minassian BA, Myers RM: Neuropathological changes in a mouse model of progressive myoclonus epilepsy: cystatin B deficiency and Unverricht-Lundborg disease. J Neuropathol Exp Neurol 2002, 61:1085-1091

29. Lehesjoki AE: Molecular background of progressive myoclonus epilepsy. EMBO J 2003, 22:3473-3478

30. Riccio M, Di Giaimo R, Pianetti S, Palmieri PP, Melli M, Santi S: Nuclear localization of cystatin B, the cathepsin inhibitor implicated in myoclonus epilepsy (EPM1). Exp Cell Res 2001, 262:84-94

31. Pawlik M, Sastre M, Calero M, Mathews PM, Schmidt SD, Nixon RA Levy E: Overexpression of human cystatin $C$ in transgenic mice does not affect levels of endogenous brain amyloid $\beta$ peptide. $J$ Mol Neurosci 2004, 22:13-18

32. Huh CG, Hakansson K, Nathanson CM, Thorgeirsson UP, Jonsson N, Grubb A, Abrahamson M, Karlsson S: Decreased metastatic spread in mice homozygous for a null allele of the cystatin C protease inhibitor gene. Mol Pathol 1999, 52:332-340

33. Mi W, Pawlik M, Sastre M, Jung SS, Radvinsky DS, Klein AM, Sommer $J$, Schmidt SD, Nixon RA, Mathews PM, Levy E: Cystatin C inhibits amyloid- $\beta$ deposition in Alzheimer's disease mouse models. Nat Genet 2007, 39:1440-1442

34. Barrett AJ, Kirschke H: Cathepsin B, cathepsin H, and cathepsin L. Methods Enzymol 1981, 80(Pt C):535-561

35. Yasuda $Y$, Kageyama T, Akamine A, Shibata M, Kominami E, Uchiyama $Y$, Yamamoto K: Characterization of new fluorogenic substrates for the rapid and sensitive assay of cathepsin $E$ and cathepsin D. J Biochem 1999, 125:1137-1143

36. Alldred MJ, Che S, Ginsberg SD: Terminal continuation (TC) RNA amplification enables expression profiling using minute RNA input obtained from mouse brain. Int J Mol Sci 2008, 9:2091-2104

37. Alldred MJ, Che S, Ginsberg SD: Terminal continuation (TC) RNA amplification without second strand synthesis. J Neurosci Methods 2009, 177:381-385

38. Ginsberg SD: Transcriptional profiling of small samples in the central nervous system. Methods Mol Biol 2008, 439:147-158

39. Mascalchi M, Michelucci R, Cosottini M, Tessa C, Lolli F, Riguzzi P, Lehesjoki AE, Tosetti M, Villari N, Tassinari CA: Brainstem involvement in Unverricht-Lundborg disease (EPM1): an MRI and (1)H MRS study. Neurology 2002, 58:1686-1689

40. Koskenkorva P, Khyuppenen J, Niskanen E, Kononen M, Bendel P, Mervaala E, Lehesjoki AE, Kalviainen R, Vanninen R: Motor cortex and thalamic atrophy in Unverricht-Lundborg disease: voxel-based morphometric study. Neurology 2009, 73:606-611

41. Riccio M, Santi S, Dembic M, Di Giaimo R, Cipollini E, CostantinoCeccarini E, Ambrosetti D, Maraldi NM, Melli M: Cell-specific expression of the epm1 (cystatin B) gene in developing rat cerebellum. Neurobiol Dis 2005, 20:104-114

42. Calkins CC, Sameni M, Koblinski J, Sloane BF, Moin K: Differential localization of cysteine protease inhibitors and a target cysteine protease, cathepsin B, by immuno-confocal microscopy. J Histochem Cytochem 1998, 46:745-751

43. Alakurtti K, Weber E, Rinne R, Theil G, de Haan GJ, Lindhout D, Salmikangas $P$, Saukko P, Lahtinen U, Lehesjoki AE: Loss of lysosomal association of cystatin B proteins representing progressive myoclonus epilepsy, EPM1, mutations. Eur J Hum Genet 2005, 13:208-215

44. Nixon RA, Yang DS, Lee JH: Neurodegenerative lysosomal disorders: a continuum from development to late age. Autophagy 2008, 4:590-599

45. Levy E, Jaskolski M, Grubb A: The role of cystatin C in cerebral 
amyloid angiopathy and stroke: cell biology and animal models. Brain Pathol 2006, 16:60-70

46. Bernstein HG, Kirschke H, Wiederanders B, Pollak KH, Zipress A, Rinne A: The possible place of cathepsins and cystatins in the puzzle of Alzheimer disease: a review. Mol Chem Neuropathol 1996, 27:225-247

47. Sun Q: Growth stimulation of 3Т3 fibroblasts by cystatin. Exp Cell Res 1989, 180:150-160

48. Tavera C, Leung-Tack J, Prevot D, Gensac MC, Martinez J, Fulcrand $P$, Colle $A$ : Cystatin $C$ secretion by rat glomerular mesangial cells: autocrine loop for in vitro growth-promoting activity. Biochem Biophys Res Commun 1992, 182:1082-1088

49. Sokol JP, Schiemann WP: Cystatin C antagonizes transforming growth factor $\beta$ signaling in normal and cancer cells. Mol Cancer Res 2004, 2:183-195

50. Selenica ML, Wang X, Ostergaard-Pedersen L, Westlind-Danielsson A, Grubb A: Cystatin C reduces the in vitro formation of soluble
A 31 -42 oligomers and protofibrils. Scand J Clin Lab Invest 2007, 67:179-190

51. Sastre M, Calero M, Pawlik M, Mathews PM, Kumar A, Danilov V Schmidt SD, Nixon RA, Frangione B, Levy E: Binding of cystatin C to Alzheimer's amyloid $\beta$ inhibits amyloid fibril formation. Neurobiol Aging 2004, 25:1033-1043

52. Kaeser SA, Herzig MC, Coomaraswamy J, Kilger E, Selenica ML, Winkler DT, Staufenbiel M, Levy E, Grubb A, Jucker M: Cystatin C modulates cerebral $\beta$-amyloidosis. Nat Genet 2007, 39:1437-1439

53. Nagai A, Ryu JK, Kobayash S, Kim SU: Cystatin C induces neuronal cell death in vivo. Ann NY Acad Sci 2002, 977:315-321

54. Ekstrom $\mathrm{U}$, Wallin $\mathrm{H}$, Lorenzo J, Holmqvist $\mathrm{B}$, Abrahamson M, Aviles FX: Internalization of cystatin C in human cell lines. FEBS J 2008 , 275:4571-4582

55. Kalviainen R, Khyuppenen J, Koskenkorva P, Eriksson K, Vanninen R, Mervaala E: Clinical picture of EPM1-Unverricht-Lundborg disease. Epilepsia 2008, 49:549-556 